prevalence of malformations among infants of mothers taking AEDs other than valproate was only $2.9 \%(\mathrm{p}<0.001)$. The relative risk of having an infant born with a major malformation for valproate-treated women is $7.3(\mathrm{p}<0.001)$.

Lamotrigine and risk of malformations. No increase in risk of major birth defects occurred in infants of mothers exposed prenatally to lamotrigine, in reports to the International Lamotrigine Pregnancy Register (ILPR) (Cunnington M, Tennis P, and the ILPR Scientific Advisory Committee. Neurology March (2 of 2) 2005;64:955-960). The risk of $2.9 \%$ with lamotrigine monotherapy was similar to that in the general population and in women exposed to other AED monotherapy (3.3-4.5\%). With lamotrigine polytherapy including valproate, the risk increased to $12.5 \%$; with polytherapy excluding valproate, the risk was $2.7 \%$.

\title{
COGNITIVE DEFICITS IN CHILDREN EXPOSED TO VALPROATE IN UTEKO
}

The long-term effects on cognitive functioning of school-aged children exposed to antiepileptic drugs (AEDs) in utero were investigated at Alder Hay Hospital and Walton Centre for Neurology and Neurosurgery, Liverpool, UK. Neuropsychological tests (WISCIII) were performed on 249 children, ages 6-16, of women with epilepsy recruited from epilepsy and obstetric clinics in the region. Children with prenatal exposure to sodium valproate (VPA) showed a significantly lower verbal IQ when compared to other AEDs or no drug exposure; their IQ was more likely to be $<69$, and memory was more often impaired. Patients taking VPA and other AEDs showed no significant differences between maternal IQ and socioeconomic status. In addition to VPA exposure, low maternal IQ and tonic-clonic seizures during pregnancy were risk factors for impaired verbal IQ in the child. Two thirds of children exposed to VPA had a verbal IQ below average and one fifth had a learning disability with a verbal IQ $<69$. Five or more tonic-clonic seizures during pregnancy were associated with impaired verbal IQ. (Vinten J, Adab N, Kini U et al. Neuropsychological effects of exposure to anticonvulsant medication in utero. Neurology March (2 of 2) 2005;64:949-954). (Reprints: Prof Gus A Baker, Dept of Clinical Neuropsychology, University Department of Neurosciences, Walton Centre for Neurology and Neurosurgery, Lower Lane, Liverpool, L9 7LJ, UK).

COMMENT. In this retrospective study controlled for maternal IQ, prenatal exposure to VPA and maternal seizures increased the risk of impairment of verbal intelligence and memory functioning in the child.

\section{FOLIC ACID-INDUCED FALL IN PHENYTOIN LEVELS AND SEIZURE RECURRENCE}

Recurrence of seizures is reported in an adult after addition of folic acid for treatment of a macrocytic anemia. He had previously been seizure free for 3 years while receiving phenytoin $300 \mathrm{mg} /$ day. After folate, the phenytoin serum level was $4.5 \mathrm{mcg} / \mathrm{mL}$; prior to the addition of $5 \mathrm{mg}$ oral folate, the phenytoin levels were consistently between 12 and 18 